\title{
The Mediating Role of Cognitive Emotion
}

\section{Regulation in BIS/BAS Sensitivities, Depression, and Anxiety Among Community-Dwelling Older Adults in China}

This article was published in the following Dove Press journal:

Psychology Research and Behavior Management

\author{
Junjun Sun (D)' \\ Yanyan Luo' \\ Hongjuan Chang' \\ Ruiqin Zhang' \\ Rui Liu' \\ Yuanyuan Jiang' \\ Huifang $X i^{2}$ \\ 'School of Nursing, Xinxiang Medical \\ University, Xinxiang, People's Republic of \\ China; ${ }^{2}$ The First Affiliated Hospital of \\ Xinxiang Medical University, Weihui, \\ People's Republic of China
}

Correspondence: Yanyan Luo School of Nursing, Xinxiang Medical University, 60I Jinsui Avenue, Hongqi District, Xinxiang 453000, People's Republic of China

Tel +86-139373I5599

Email139373I5599@163.com
Background: The behavioral inhibition system (BIS) and behavioral activation system (BAS), which primarily underlie emotions and behaviors, are associated with depression and anxiety. However, the reasons behind these associations require further exploration.

Objective: This study aims to examine the mediating effects of cognitive emotion regulation between BIS/BAS and depression/anxiety among community-dwelling elderly Chinese. Methods: A cross-sectional survey was conducted with a sample of 836 elderly individuals. Structural equation modeling was used to determine relationships among BIS/BAS, cognitive emotion regulation, and depression/anxiety.

Results: Participants reporting higher BIS sensitivity were more likely to use maladaptive cognitive emotion regulation strategies, which were in turn associated with higher rates of depression and anxiety. BAS sensitivity was more likely to lead to adaptive cognitive emotion regulation strategies, which resulted in lower levels of depression and anxiety.

Conclusion: Our findings suggest that incorporating emotional regulation in interventions targeting BIS/BAS sensitivities may enhance the accuracy and efficiency of these treatments for depression and anxiety.

Keywords: behavioral inhibition system, behavioral activation system, cognitive emotion regulation, depression, anxiety

\section{Introduction}

Optimizing mental health provides important support for healthy aging, ${ }^{1}$ since depression and anxiety are common in elderly adults and place a heavy burden on families and society. A recent community-based survey showed that personality is strongly linked to anxiety and depression in later life. ${ }^{2}$ Most contemporary personality studies are based on static trait-oriented models, such as the Big Five. However, trait-based personality models cannot account for internal mental mechanisms. Tools such as Gray's reinforcement sensitivity theory (RST) provide a more detailed method to understand personality based on neurobiology, ${ }^{3}$ and this model has been an effective framework for researching the association between personality and mood disorders. ${ }^{4}$

According to RST, two primary motivational systems underlie human emotions and behaviors: the behavioral inhibition system (BIS) and the behavioral activation 
system (BAS), which are measured using the BIS/BAS Scale. ${ }^{5}$ These two systems illustrate how changes in personal moods and behaviors occur based on different background factors. An excessively sensitive BIS causes increased avoidance behavior and negative emotions in response to punishments or threats. By contrast, an undersensitive BAS causes a lack of positive experiences and reduced positive emotions due to decreased approach movement. ${ }^{6,7}$ Individual differences in BAS and BIS sensitivities lead to differences in appetitive processing, aversive processing, and personality. Individuals with a more sensitive BAS tend to exhibit more cognitive styles and emotions associated with rewarding stimuli and extraversion personality, ${ }^{4,8}$ whereas a more sensitive BIS impacts behaviors and psychological processes related to punishment and neuroticism. ${ }^{9}$

The RST cognitive model of psychopathology posits that individuals with dysregulated levels of BIS and BIS sensitivity are at increased risk for subsequent psychopathology. ${ }^{4,10}$ Studies have found that depression is negatively associated with BAS sensitivity and either positively or not associated with BIS sensitivity ${ }^{11}$ Increased BIS sensitivity has also been found to be a risk factor for affective disorders in general, with possibly a slightly more pronounced role in anxiety than depression. ${ }^{12}$ These inconsistent results may be explained by BIS being a state-dependent characteristic of depression while BAS represents a trait-vulnerability index. ${ }^{4}$ Anxiety, meanwhile, is consistently associated with high BIS sensitivity but only weakly, or not at all, associated with low BAS sensitivity. ${ }^{13}$ A recent meta-analysis found that BIS is a higher-order, shared factor for both depression and anxiety, whereas BAS is specific to depression. ${ }^{14}$ In sum, extensive research has found that dysregulated BIS/BAS sensitivities play a critical roles in the development and maintenance of depression and anxiety. ${ }^{15}$ However, to the best of our knowledge, relevant research is rarely conducted among older adults. Therefore, it is of interest to investigate the relationship between BIS/BAS and depression/anxiety among community-dwelling elderly.

Furthermore, the mechanisms between BIS/BAS sensitivities and depression/anxiety remain unclear. Individuals with similar personality traits can follow different development pathways due to moderating factors. The developmental psychopathology perspective emphasizes the importance of self-regulatory that enable individuals to modulate emotional responses and thereby control the risk associated with such reactions. ${ }^{3,16}$ Studies suggest that BIS/BAS can be understood as mental mechanisms contributing to an individual's ability to self-regulate, including depression and anxiety. ${ }^{17}$ Emotional regulation is an important form of self-regulation, ${ }^{18}$ and evidence suggests that dysregulated BIS/BAS sensitivities can negatively affect this type of self-regulation. ${ }^{19}$

Studies show that BIS/BAS sensitivities impact individuals' emotional regulation, thereby increasing the likelihood of depression and anxiety. For example, one study reveals that difficulties in emotional regulation mediate the association between BIS/BAS activation and depression and anxiety among college students. ${ }^{20}$ Another suggests that two commonly used emotional strategies - expressive suppression and cognitive reappraisal-partially mediate the association between BIS activation and negative effects, but find no correlation between BAS activation and these strategies. ${ }^{21}$ However, BAS sensitivity may affect depression only under certain conditions such as low cognitive reappraisal scores. ${ }^{22} \mathrm{~A}$ recent longitudinal study of German adolescents shows that high BAS sensitivity can predict higher adaptive emotional strategies. ${ }^{23}$ Similarly, people with high BAS scores are more likely to regulate their emotions and behaviors effectively. ${ }^{24,25}$ Meanwhile, low BAS sensitivity and high BIS sensitivity are related to emotion regulation difficulties. ${ }^{26}$

However, this preexisting literature on the association between BIS/BAS sensitivity and negative affect has mostly focused on certain emotion regulation strategies or emotion regulation difficulties, ${ }^{27}$ while the cognitive emotion regulation which consist of adaptive and maladaptive strategies seems to be strongly linked to depression and anxiety. ${ }^{17}$ Furthermore, most previous studies focus on adolescents or university students, while few examine the associations between BIS/BAS and negative emotions among older adults.

Accordingly, this study attempts to better characterize the relationship between BIS/BAS sensitivities, depression, and anxiety among older adults dwelling in the community. We opted to use the seven-item Generalized Anxiety Disorder Scale (GAD-7) ${ }^{28}$ to measure anxiety, considering that generalized anxiety disorder has a relationship with BIS. ${ }^{13}$ We hypothesize that BAS sensitivity is negatively associated with depression and anxiety, and that these effects are at least partially mediated by the positive relationship between BAS and adaptive cognitive emotion regulation strategies. We also hypothesize that BIS sensitivity is positively associated with 
depression and anxiety, and that these effects are mediated by maladaptive cognitive emotion regulation strategies. To test these hypotheses, we use structural equation modeling (SEM) to examine the mediating role of cognitive emotion regulation in the above relationships, classifying cognitive emotion regulation strategies as adaptive or maladaptive based on their consequences. $^{17}$

\section{Materials and Methods}

\section{Participants}

This cross-sectional study recruited older adults who resided in communities. Trained interviewers collected questionnaire data through face-to-face, one-on-one interviews. Participants were all 65 years or older, and were able to walk independently. We excluded respondents with poor hearing or eyesight and those diagnosed with dementia. A total of 850 older adults completed a questionnaire survey consisting of the BAS/BIS Scale, the short version of the Cognitive Emotion Regulation Questionnaire (CERQ-short) ${ }^{29}$ the 15 -item Geriatric Depression Scale (GDS-15), ${ }^{30}$ and the seven-item GAD-7. We excluded 14 individuals due to missing data, leaving a final sample size of 836 .

\section{Ethical Statements}

The study, including the questionnaire, was approved by the Medical Ethics Committee of Xinxiang Medical University (2019-HLPY-A001) and in accordance with the Declaration of Helsinki. The study was performed according to approved guidelines, and all volunteers signed to confirm their informed consent for participation. Throughout the study, issues related to the safety and wellbeing of participants and data privacy were monitored. Participants scoring 5 or higher on the GDS-15 or GAD-7 were referred to a mental healthcare professional for further psychological evaluation.

\section{Measurements BIS/BAS Scale}

We used the BIS/BAS Scale ${ }^{5}$ to assess individual sensitivities to punishment and reward. This scale contains 20 items, each of which are rated on a 4-point Likert scale ranging from 1 ("strongly disagree") to 4 ("strongly agree"). These 20 items comprise two subscales: the BIS scale includes 7 items and the BAS scale 13 items. The BAS subscale can be further divided into BAS-Drive (BAS-D), BAS-Fun Seeking (BAS-F), and BAS-Reward Responsiveness (BAS-R).
Studies have found the Chinese version of the BIS/BAS Scale to have sufficient validity and reliability. ${ }^{31}$ In the current study, the Cronbach's $\alpha$ values for the overall scale and for the BAS and BIS subscales were 0.80, 0.80, and 0.70 , respectively. The three BAS subscales had lower internal consistencies (Cronbach's $\alpha$ ranged from 0.55-0.72).

\section{CERQ-Short}

The CERQ-short ${ }^{29}$ is an 18-item self-reported assessment of multiple cognitive emotion regulation strategies in response to stressful life events. The instrument includes nine conceptually distinct strategies, divided into adaptive (acceptance, refocusing on planning, positive refocusing, positive reappraisal, and putting into perspective) and maladaptive strategies (self-blame, blaming others, rumination, and catastrophizing). Participants rate how often they use each strategy on a 5-point Likert scale ranging from 1 ("almost never") to 5 ("almost always"). Higher scores on each strategy denote greater frequency of use.

The Chinese version of the CERQ-short shows acceptable reliability and validity. ${ }^{32}$ In the current study, the Cronbach's $\alpha$ values of the positive and negative subscales were 0.79 and 0.73 , respectively.

\section{GDS-15}

The GDS- $15^{30}$ is commonly used to screen for depression among older people. This self-reported scale includes 15 items answered using a simple response format: "yes" (1) or "no" (0). Scores below 5 indicate minimal depression, and scores $\geq 5$ indicate depression. Participants with higher scores are considered to have more severe depression. The Chinese version of the GDS-15 has been proven efficient. ${ }^{33}$ The scale's Cronbach's $\alpha$ in this study was 0.76 .

\section{GAD-7}

The GAD- $7^{28}$ is a seven-item scale focused on screening for anxiety symptoms. The items are rated on a 4-point Likert scale ranging from 0 ("not at all") to 3 ("nearly every day"). Scores below 5 indicate minimal anxiety, and scores $\geq 5$ indicate anxiety. The higher the score, the more severe the symptoms. The Chinese version of GAD-7 has good reliability and validity. In the current study, the scale's Cronbach's $\alpha$ was 0.91 .

\section{Demographic Information}

Demographic variables included gender, age, education, residential status, and occupation before retirement. 


\section{Statistical Analysis}

We calculated descriptive statistics to describe respondents' sociodemographic characteristics and performed Pearson correlation analyses to examine the associations between study variables.

We tested the mediation model using SEM with BIS and BAS scores as the independent variable, GDS and GAD scores as the dependent variable, and the two latent variables CERQmaladaptive and CERQ-adaptive as the mediating variables. Gender and age were used as covariates in each mediation analysis. All analyses were conducted using the maximum likelihood estimation method. We used several goodness-offit indices to evaluate model fit: $\chi^{2}, \chi^{2} / \mathrm{df}$, root mean square error of approximation (RMSEA), comparative fit index (CFI), standardized root mean square residual (SRMR), goodness-offit index (GFI), and adjusted goodness-of-fit index (AGFI).

Generally, well-fitting models are indicated when RMSEA $<0.06, \mathrm{CFI}>0.09, \mathrm{SRMR}<0.08, \mathrm{GFI}>0.09$, and AGFI $>$ $0.09 .{ }^{34} \mathrm{~A}$ value of $\chi^{2} / \mathrm{df}$ between 0 and 3 indicates that the model has an acceptable fit. ${ }^{35} \mathrm{Chi}$-square tends to reject models based on large sample sizes, so it was not reported as a goodness-of-fit criterion in the current study. To test the mediation effect, the bias-corrected $95 \%$ confidence interval (CI) was calculated with 1000 bootstrapping samples. If the 95\% CI of the indirect effect does not include 0 , a significant mediation effect can be established. All analyses were conducted in SPSS 22.0 and AMOS 22.0.

\section{Results}

\section{Characteristics of the Study Sample}

Table 1 presents participant demographics and descriptive statistics for all study variables. The participants' mean age was 70.99 ( \pm 5.59$)$ years, and 407 (48.68\%) participants were female. Of the 836 participants, 294 (35.17\%) reported symptoms of depression (GDS-15) and 204 (24.5\%) reported symptoms of anxiety (GAD-7).

\section{Correlations Between Variables}

Table 2 shows the correlation matrix, skewness, and kurtosis for the study variables. All variables met the criteria of normality, with skewness below the absolute value of 3 $(-0.413$ to 1.868$)$ and kurtosis below the absolute value of $8(-0.350$ to 3.232$) .^{36}$ There was no multicollinearity in the correlations between variables. All associations were statistically significant, with the exception of those between BIS and CERQ-adaptive $(\mathrm{r}=-0.024, p=$ 0.552), BAS and CERQ-maladaptive $(\mathrm{r}=0.068, p=$
Table I Characteristics of the Study Sample

\begin{tabular}{|c|c|}
\hline Characteristics & Total Sample $(\mathbf{N}=\mathbf{8 3 6})$ \\
\hline Female, n (\%) & $407(48.68)$ \\
\hline Age, mean (SD) [range] & $70.99(5.59)$ [65-90] \\
\hline \multicolumn{2}{|l|}{ Educational level, n (\%) } \\
\hline Primary education & $14 \mid(\mid 6.87)$ \\
\hline Secondary education & $269(32.18)$ \\
\hline High school or above & $426(51.95)$ \\
\hline \multicolumn{2}{|l|}{ Marital status, n (\%) } \\
\hline Married & $684(81.82)$ \\
\hline Divorced & $2 \mathrm{I}(2.5 \mathrm{I})$ \\
\hline Widowed & $13 \mid(\mid 5.67)$ \\
\hline BIS, mean (SD) [range] & $13.42(3.28)[5-20]$ \\
\hline BAS, mean (SD) [range] & $40.30(6.21)[19-52]$ \\
\hline CERQ-maladaptive, mean (SD) [range] & $17.98(5.09)[8-40]$ \\
\hline CERQ-adaptive, mean (SD) [range] & $30.06(6.90)[13-50]$ \\
\hline \multicolumn{2}{|l|}{ Depression (GDS-15), n (\%) } \\
\hline$\geq 5$ & $294(35.17)$ \\
\hline$<5$ & $542(64.83)$ \\
\hline \multicolumn{2}{|l|}{ Anxiety (GAD-7), n (\%) } \\
\hline$\geq 5$ & $204(24.50)$ \\
\hline$<5$ & $631(75.50)$ \\
\hline
\end{tabular}

Abbreviations: BIS, behavioral inhibition system; BAS, behavioral activation system; CERQ-maladaptive, maladaptive cognitive emotion regulation strategies; CERQ-adaptive, adaptive cognitive emotion regulation strategies; GDS-15, I5item Geriatric Depression Scale; GAD-7, Generalized Anxiety Disorder Scale.

0.095), and BAS and anxiety $(\mathrm{r}=-0.058, p=0.156)$. However, it should be noted that the correlation values were low. The descriptive data and the existing correlations in the subdimensions of all the study variables are provided in the Supplementary Table-1.

\section{Model Fit and Parameter Estimates: Revised Structural Model}

According to the modification indices, the initial model of the relationships between BIS/BAS and depression was altered to improve fit. We added several covariance terms between the BAS-F and BAS-D error terms: acceptance and putting into perspective and positive refocusing and putting into perspective, respectively. Figure 1 shows the final model of the relationships between BIS/BAS and depression (GDS-15), as mediated by maladaptive and adaptive cognitive emotion regulation strategies. The results show that the model has acceptable fit indices: $\chi^{2}$ 
Table 2 Correlation Matrix, Skewness, and Kurtosis for the Study Variables

\begin{tabular}{|l|l|l|l|l|l|l|}
\hline Variables & $\mathbf{I}$ & $\mathbf{2}$ & $\mathbf{3}$ & $\mathbf{4}$ & $\mathbf{5}$ \\
\hline I.BIS & - & & & & \\
\hline 2.BAS & $0.266^{* *}$ & - & & & \\
\hline 3.CERQ-maladaptive & $0.394^{* *}$ & 0.068 & - & & \\
\hline 4. CERQ-adaptive & -0.024 & $0.167^{* *}$ & $0.200^{* *}$ & - & \\
\hline 5. GDS-15 & $0.238^{* *}$ & $-0.183^{* *}$ & $0.353^{* *}$ & $-0.211^{* *}$ & - & \\
\hline $6 . G A D-7$ & $0.314^{* *}$ & -0.058 & $0.426^{* *}$ & $-0.095^{*}$ & $0.599 * *$ \\
\hline Skewness & -0.246 & -0.413 & 0.694 & 0.418 & - \\
\hline Kurtosis & -0.350 & -0.148 & 1.283 & -0.097 & 0.879 \\
\hline
\end{tabular}

Notes: $* p<0.05 ; * * p<0.01$

Abbreviations: BIS, behavioral inhibition system; BAS, behavioral activation system; CERQ-maladaptive, maladaptive cognitive emotion regulation strategies; CERQadaptive, adaptive cognitive emotion regulation strategies; GDS-15, 15-item Geriatric Depression Scale; GAD-7, Generalized Anxiety Disorder Scale.

$=219.917(d f=80, \mathrm{p}<0.001), \chi^{2} / \mathrm{df}=2.749, \mathrm{GFI}=0.953$, $\mathrm{CFI}=0.931, \mathrm{AGFI}=0.929, \mathrm{SRMR}=0.064$, and RMSEA $=0.054(90 \%$ CI: $0.046,0.063)$.

We also altered the initial model of the relationship between BIS/BAS and anxiety by adding several covariance terms between the self-blame and rumination error terms: acceptance and putting into perspective and positive refocusing and putting into perspective, respectively. Figure 2 shows the final model of the relationships between BIS/BAS and anxiety (GAD-7), as mediated by maladaptive and adaptive cognitive emotion regulation strategies. The model has acceptable fit indices: $\chi^{2}=$ $228.541(d f=80, \mathrm{p}<0.001), \chi^{2} / \mathrm{df}=2.857$, GFI $=$ 0.952 , CFI $=0.927$, AGFI $=0.927$, SRMR $=0.066$, and RMSEA $=0.056$ (90\% CI: $0.047,0.064)$.

\section{Direct and Indirect Effects}

As shown in Figure 1, all path coefficients are significant in the depression model. We found that BIS directly affects adaptive and maladaptive cognitive emotion

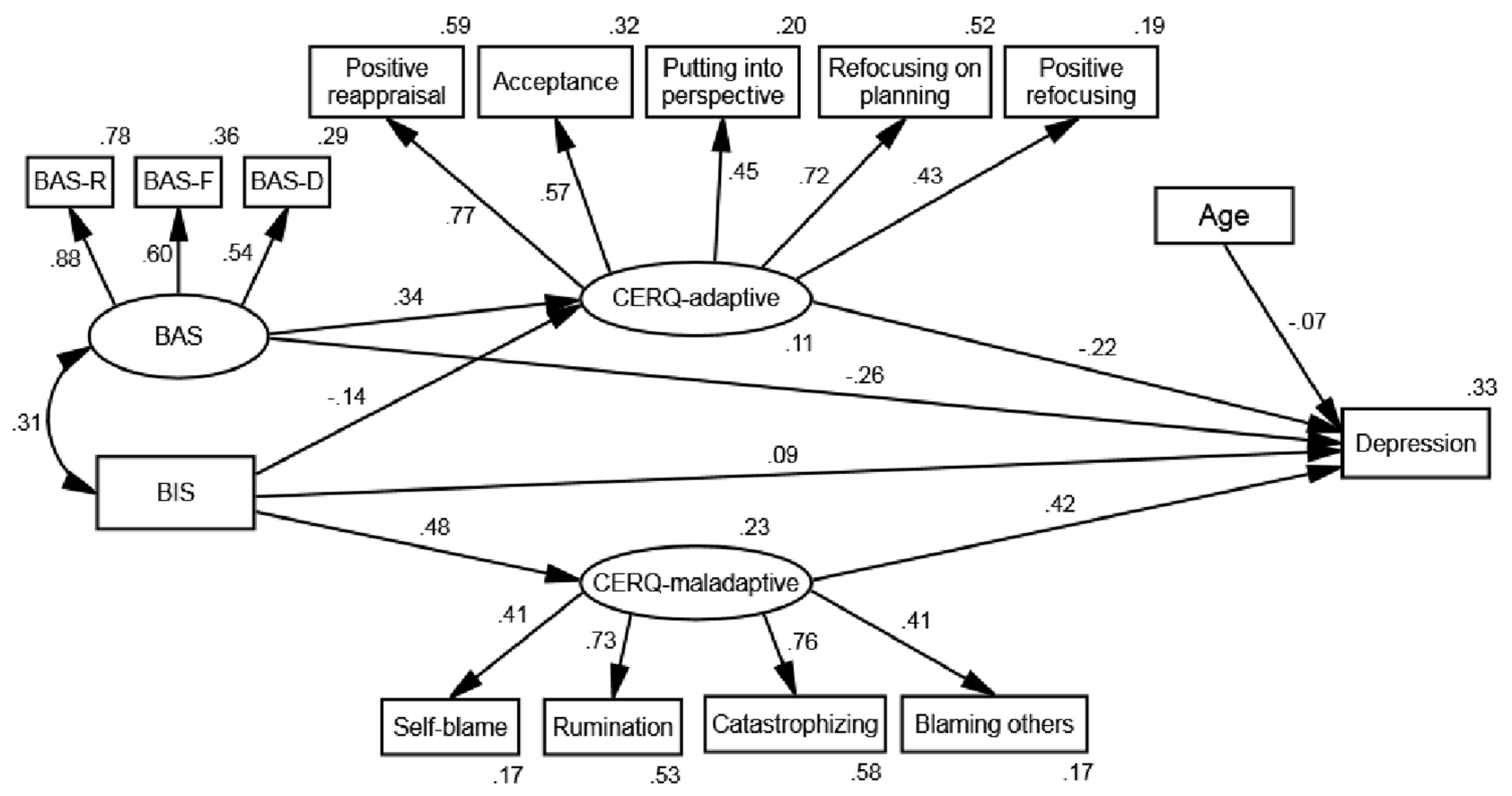

Figure I Mediation of the relationship between BIS/BAS and depression through CERQ-adaptive and CERQ-maladaptive. Path coefficients were standardized. Observed variables are represented by ovals and latent variables by rectangles. 


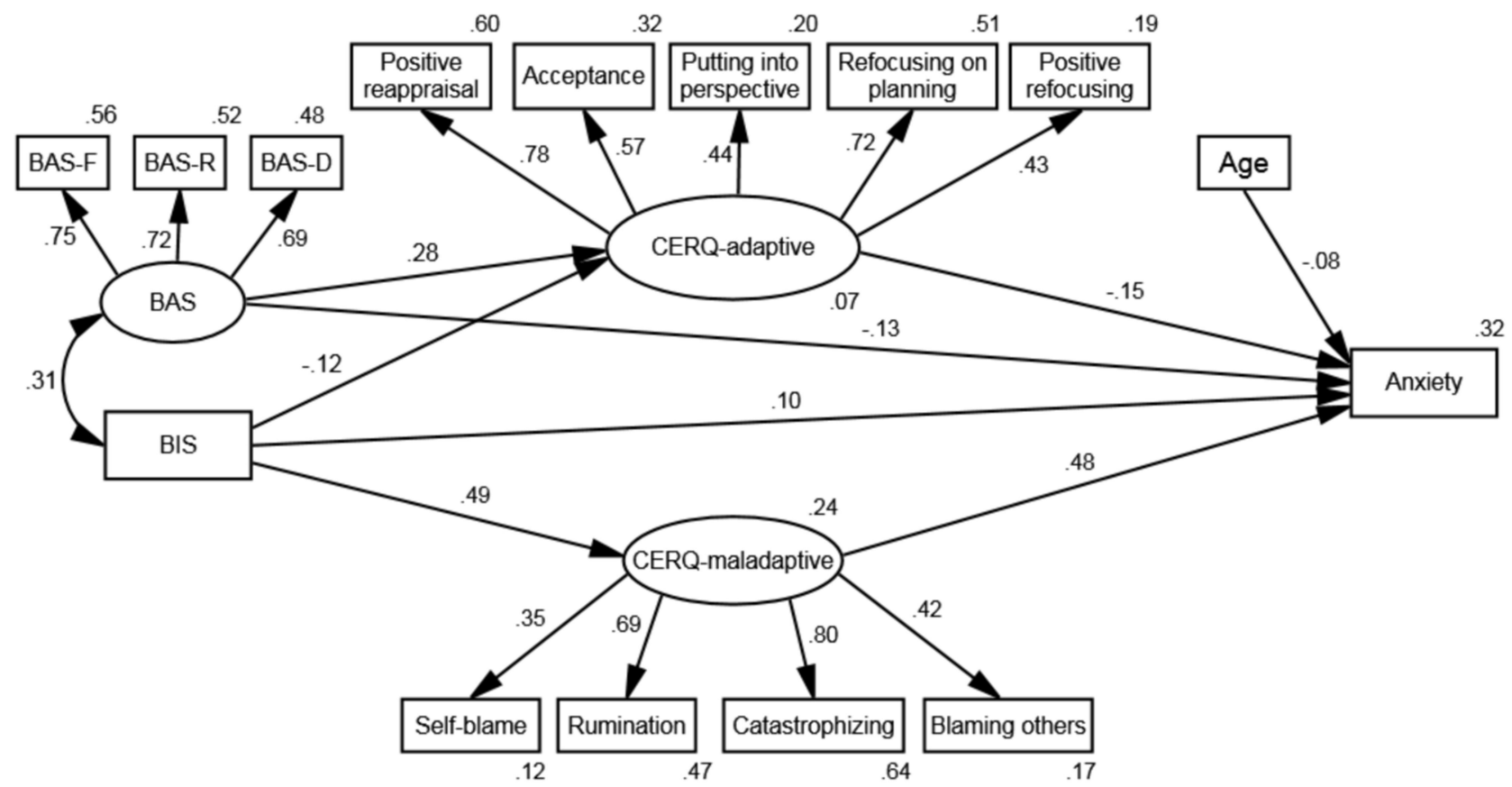

Figure 2 Mediation of the relationship between BIS/BAS and anxiety through CERQ-adaptive and CERQ-maladaptive. Path coefficients were standardized. Observed variables are represented by ovals and latent variables by rectangles.

regulation strategies, both of which directly affect depression. The direct path from BIS to depression is significant $(\beta=0.094, p<0.05)$, suggesting that the relationship between BIS and depression is partially mediated by adaptive and maladaptive emotion regulation strategies. We also found that BAS directly affects adaptive cognitive emotion regulation strategies, which directly affect depression. The direct path from BAS to depression is significant $(\beta=-0.256, p<0.01)$, suggesting that the relationship between BAS and depression is partially mediated by adaptive emotion regulation strategies. Lastly, age is significantly negatively related to depression $(\beta=-0.074, \mathrm{p}<0.01)$, whereas gender is not.

As shown in Figure 2, all path coefficients are significant in the anxiety model. We found that BIS directly affects adaptive and maladaptive cognitive emotion regulation strategies, both of which directly affect anxiety. The direct path from BIS to anxiety is significant $(\beta=0.100$, $\mathrm{p}<0.05$ ), indicating that the relationship between BIS and anxiety is partially mediated by adaptive and maladaptive emotion regulation strategies. BAS also directly affects adaptive cognitive emotion regulation strategies, which directly affect anxiety. The direct path from BAS to anxiety is significant $(\beta=-0.127, p<0.01)$, indicating that the relationship between BAS and anxiety is partially mediated by adaptive emotion regulation strategies.
Lastly, age is significantly negatively related to anxiety ( $\beta=-0.079, p<0.01)$, whereas gender is not.

In general, both adaptive and maladaptive cognitive emotion regulation strategies partially mediate the associations of BIS with depression and anxiety. However, only adaptive cognitive emotion regulation strategies partially mediate the associations of BAS with depression and anxiety. Table 3 presents the results of bootstrapping, which show that all the model's indirect effects are statistically significant.

\section{Discussion}

The present study examines the associations between BIS/ BAS sensitivities and depression and anxiety, together with the mediating roles of adaptive and maladaptive cognitive emotion regulation strategies, among older Chinese adults who live in communities. To the best of our knowledge, this is the first investigation of these associations among this population.

Our results make several important contributions. First, we found that older people reporting high BIS sensitivity tended to use more maladaptive cognitive emotion regulation strategies, which were associated with higher rates of depression and anxiety. This proves that maladaptive cognitive emotion regulation strategies may underlie the negative effect of behavioral inhibitions on severe depression 
Table 3 Direct and Indirect Effects of the Two Final Models

\begin{tabular}{|l|l|l|l|l|l|}
\hline Effect & Path & Estimate & P-value & \multicolumn{2}{l|}{ 95\% Bias-Corrected CI } \\
\hline \multirow{2}{*}{ Direct } & BAS $\rightarrow$ Depression & -0.256 & 0.001 & -0.351 & -0.169 \\
& BIS $\rightarrow$ Depression & 0.094 & 0.036 & 0.007 & 0.194 \\
& BAS $\rightarrow$ Anxiety & -0.127 & 0.008 & -0.211 & -0.037 \\
& BIS $\rightarrow$ Anxiety & 0.100 & 0.040 & 0.005 & 0.188 \\
\hline \multirow{2}{*}{ Indirect } & BAS $\rightarrow$ CERQ-adaptive $\rightarrow$ Depression & -0.073 & 0.002 & -0.114 & -0.041 \\
& BIS $\rightarrow$ CERQ-adaptive $\rightarrow$ Depression & 0.030 & 0.002 & 0.007 & 0.058 \\
& BIS $\rightarrow$ CERQ-maladaptive $\rightarrow$ Depression & 0.202 & 0.002 & 0.109 & 0.277 \\
& BAS $\rightarrow$ CERQ-adaptive $\rightarrow$ Anxiety & -0.040 & $<0.001$ & -0.073 & -0.014 \\
& BIS $\rightarrow$ CERQ-adaptive $\rightarrow$ Anxiety & 0.018 & $<0.001$ & 0.004 & 0.057 \\
& BIS $\rightarrow$ CERQ-maladaptive $\rightarrow$ Anxiety & 0.235 & $<0.001$ & 0.221 & 0.434 \\
\hline
\end{tabular}

Abbreviations: BIS, behavioral inhibition system; BAS, behavioral activation system; CERQ-maladaptive, maladaptive cognitive emotion regulation strategies; CERQadaptive, adaptive cognitive emotion regulation strategies.

and anxiety symptoms. These findings are consistent with those of prior studies reporting that BIS sensitivity is positively associated with emotion regulation difficulties in undergraduates, ${ }^{20}$ and that maladaptive cognitive emotion regulation strategies mediate the relationship between BIS sensitivity and anxiety. ${ }^{23}$ According to RST, high BIS sensitivity induces behavioral inhibition, which causes avoidance.$^{37}$ Individuals with higher BIS scores are, therefore, more likely to focus on potential threats and have inhibited awareness or regulation of their emotions. This means that elderly people with higher BIS sensitivity are more likely to choose maladaptive strategies. According to cognitive behavioral theory, maladaptive cognitive appraisal of stressful events may be at the core of depression and anxiety: ${ }^{38}$ a recent meta-analytic review suggests that both depression and anxiety show strong positive associations with avoidance and rumination, which are both maladaptive emotion regulation strategies. ${ }^{16}$

Second, we found that high BAS sensitivity leads to lower levels of depression and anxiety and enhanced adaptive cognitive emotion regulation strategies. These findings are in line with those of previous studies reporting that BAS sensitivity is negatively associated with negative affect ${ }^{24}$ and can positively predict adaptive cognitive emotion regulation. ${ }^{23}$ BAS drives elderly adults to achieve goals, leading to effective implementation of adaptive strategies and a feeling of enjoyment when they attain them. However, our results are inconsistent with those of studies on adults with chronic pain, which find no association between BAS and emotional regulation. ${ }^{21}$ One possible explanation for this inconsistency is that individuals in pain may find it difficult to perform adaptive cognitive emotion regulation strategies, and thus may show weaker relationships between BAS and adaptive strategies. ${ }^{39}$ Further, socioemotional selectivity theory states that older people can more effectively adjust their emotions, ${ }^{40}$ which may suggest that those with higher BAS sensitivity are more apt to implement adaptive strategies effectively. Given that adolescents with higher BAS tend to score higher in aggressive behaviors, ${ }^{41}$ further investigation is needed into why we did not find the same for older people. In addition, age differences in depression and anxiety were found in the current study: specifically, younger elderly had higher levels of depression and anxiety, which may be explained by younger elderly having higher expectations for future life.

Third, both of our final models revealed a negative link between BIS and adaptive strategies that we had not hypothesized, suggesting that adaptive strategies significantly mediate the relationships of BIS with depression and anxiety. This novel finding contrasts with the outcomes of previous studies among adolescents, which have found no significant links between BIS and the adaptive strategy of cognitive reappraisal. ${ }^{23}$ However, studies have found positive relationships between BIS and catastrophizing and rumination, ${ }^{27,31}$ which are both characterized by a focus on negative and terrifying thoughts associated with an event. ${ }^{42}$ One previous study suggests that the association between BIS and negative affect is mediated by expressive suppression. ${ }^{21}$ These findings are in line with our results. One possible explanation for our surprising finding is that the behavioral inhibition induced by BIS may suppress elderly people's ability to face their thoughts, thus making adaptive strategies more difficult. In any event, additional research is needed to determine if this unexpected result can be replicated. 


\section{Limitations and Conclusions}

The present study had several main limitations. First, owing to its cross-sectional design it cannot evaluate casual relationships between variables; a longitudinal design is necessary to serially assess changes in variables. Second, all variables were assessed using self-report methods, meaning that the strength of associations may have been artificially increased by common method bias. It would be interesting to measure the variables using other assessment methods, such as ecological momentary assessment. Third, this study applied Carver and White's BIS/BAS Scale, which does not measure BIS and fight-flightfreeze System (FFFS) separately. Considering that current RST research assesses these two systems individually, future studies should apply the revised RST to demonstrate whether BIS and FFFS have different effects on emotion regulation strategies. Fourth, although the BIS/BAS scale showed acceptable levels of reliability and validity, some activities or examples in the items were difficult to relate to older adults' actual living conditions and so may not facilitate identification. Finally, we only evaluated symptoms of generalized anxiety disorder, whereas many older adults have other types of anxiety disorders, which should be considered in future research.

Despite these limitations, this study has several strengths, including the large sample of communitydwelling elderly in China and the use of SEM. Moreover, the findings substantially elucidate the involvement of two motivational systems, namely BIS and BAS, in depression and anxiety, and how these relationships are mediated by adaptive and maladaptive cognitive emotion regulation strategies. Accordingly, although BIS/BAS sensitivities may be the focus of interventions for negative emotions, our study suggests that adding emotion regulation could enhance the accuracy and efficiency of these practical applications.

\section{Implications for Practice}

In this study, the proportions of community-dwelling elderly with symptoms suggestive of depression and anxiety were $35.2 \%$ and $24.5 \%$, respectively. Higher prevalence of these symptoms among the elderly indicates that mental health professionals should be encouraged to screen for such symptoms within Chinese communities. Our results indicate that older people with high BIS sensitivity are more likely to use maladaptive cognitive emotion regulation strategies, which are associated with higher rates of depression and anxiety. However, high BAS sensitivity is associated with adaptive cognitive emotion regulation strategies, which reduce depression and anxiety. These findings suggest that mental health professionals should focus on low BAS and/or high BIS as high risk factors for depression and anxiety among older people. Treatment plans should include teaching them more adaptive cognitive emotion regulation strategies, and reducing maladaptive cognitive emotion regulation strategies. The present study provides community workers with valuable guidance for screening and reducing depression and anxiety symptoms among older adults.

\section{Funding}

This research was funded by the Key Scientific Research Projects Plan of Henan Higher Education Institutions (19B320014) and the Humanities and Social Science Project of Henan Education Department (2020-ZZJH $-374)$.

\section{Disclosure}

The authors report no conflicts of interest in this work.

\section{References}

1. National Prevention Council. Healthy Aging in Action: Advancing the National Prevention Strategy; 2016.

2. Gale C, Sayer AA, Cooper C, et al. Factors associated with symptoms of anxiety and depression in five cohorts of community-based older people: the HALCyon (Healthy Ageing across the Life Course) programme. Psychol Med. 2011;41(10):2057-2073. doi:10.1017/ S0033291711000195

3. Collins MD, Jackson CJ, Walker BR, O'Connor PJ, Gardiner E. Integrating the context-appropriate balanced attention model and reinforcement sensitivity theory: towards a domain-general personality process model. Psychol Bull. 2017;143(1):91-106. doi:10.1037/ bul0000082

4. Bijttebier P, Beck I, Claes L, Vandereycken W. Gray's Reinforcement Sensitivity Theory as a framework for research on personality-psychopathology associations. Annu Rev Clin Psychol. 2009;29 (5):421-430. doi:10.1016/j.cpr.2009.04.002

5. Carver CS, White TL. Behavioral inhibition, behavioral activation, and affective responses to impending reward and punishment: the BIS/ BAS scales. J Pers Soc Psychol. 1994;67(2):319-333. doi:10.1037/ 0022-3514.67.2.319

6. Gray JA. Perspectives on anxiety and impulsivity: a commentary. J Res Pers. 1987;21(4):493-509. doi:10.1001/jama.2016.10486

7. Olino TM, Mcmakin DL, Forbes EE. Toward an empirical multidimensional structure of anhedonia, reward sensitivity, and positive emotionality: an exploratory factor analytic study. Assess. 2018;25 (6):679-690. doi:10.1177/1073191116680291

8. Corr PJ, Cooper AJ. The Reinforcement Sensitivity Theory of Personality Questionnaire (RST-PQ): development and validation. Psychol Assess. 2016;28(11):1427-1440. doi:10.1037/pas0000273

9. Smillie LD, Jackson CJ, Dalgleish LI. Conceptual distinctions among Carver and White's (1994) BAS scales: a reward-reactivity versus trait impulsivity perspective. Pers Individ Dif. 2006;40(5):1039-1050. doi:10.1016/j.paid.2005.10.012 
10. Corr P. The reinforcement sensitivity theory of personality Int $J \quad$ Psychophysiol. 2008;69(3):151-152. doi:10.1016/j. ijpsycho.2008.05.377

11. Segarra P, Ross SR, Pastor MC, et al. MMPI-2 predictors of Gray's two-factor reinforcement sensitivity theory. Pers Individ Dif. 2007;43 (3):437-448. doi:10.1016/j.paid.2006.12.013

12. Struijs SY, Lamers F, Rinck M, et al. The predictive value of approach and avoidance tendencies on the onset and course of depression and anxiety disorders. Depress Anxiety. 2018;35 (6):551-559. doi:10.1002/da.22760

13. Kramer SL, Rodriguez BF. A comparison of reinforcement sensitivity theory measures: unique associations with social interaction anxiety and social observation anxiety. Assessment. 2018;25(5):627-639. doi:10.1177/1073191116654003

14. Katz BA, Matanky K, Aviram G, Yovel I. Reinforcement sensitivity, depression and anxiety: a meta-analysis and meta-analytic structural equation model. Clin Psychol Rev. 2020;77:101842. doi:10.1016/j. cpr.2020.101842

15. Arfaie A, Safikhanlou S, Bakhshipour Roodsari A, Farnam A, Shafiee-Kandjani AR. Assessment of behavioral approach and behavioral inhibition systems in mood disorders. Basic Clin Neurosci. 2018;9(4):261-268. doi:10.32598/bcn.9.4.261

16. Schäfer JZ, Naumann E, Holmes EA, Tuschen-Caffier B, Samson AC. Emotion regulation strategies in depressive and anxiety symptoms in youth: a meta-analytic review. $J$ Youth Adolesc. 2017;46 (2):261-276. doi:10.1007/s10964-016-0585-0

17. Ewa D, Magorzata F. Cognitive emotion regulation strategies in anxiety and depression understood as types of personality. Front Psychol. 2018;9:856. doi:10.3389/fpsyg.2018.00856

18. Sheppes G, Suri G, Gross JJ. Emotion regulation and psychopathology. Annu Rev Clin Psychol. 2015;11(1):379-405. doi:10.1146/annurev-clinpsy-032814-112739

19. Tull MT, Gratz KL, Latzman RD, Kimbrel NA, Lejuez CW. Reinforcement sensitivity theory and emotion regulation difficulties: a multimodal investigation. Pers Individ Dif. 2010;49(8):989-994. doi:10.1016/j.paid.2010.08.010

20. Markarian SA, Pickett SM, Deveson DF, Kanona BB. A model of BIS/BAS sensitivity, emotion regulation difficulties, and depression, anxiety, and stress symptoms in relation to sleep quality. Psychiatry Res. 2013;210(1):281-286. doi:10.1016/j.psychres.2013.06.004

21. Serrano-Ibáñez ER, Ramírez-Maestre C, López-Martínez AE, et al. Behavioral inhibition and activation systems, and emotional regulation in individuals with chronic musculoskeletal pain. Front Psychiatry. 2018:9. doi:10.3389/fpsyt.2018.00394.

22. Dennis TA. Interactions between emotion regulation strategies and affective style: implications for trait anxiety versus depressed mood. Motiv Emot. 2007;31(3):200-207. doi:10.1007/s11031-007-9069-6

23. Izadpanah S, Schumacher M, Bähr A, et al. A 5-year longitudinal study of the adolescent reinforcement sensitivity as a risk factor for anxiety symptoms in adulthood: investigating the indirect effect of cognitive emotion regulation. Pers Individ Dif. 2016;95:68-73. doi:10.1016/j.paid.2016.02.021

24. Merchan-Clavellino A, Alameda-Bailen JR, Zayas Garcia A, Guil R. Mediating effect of trait emotional intelligence between the Behavioral Activation System (BAS)/Behavioral Inhibition System (BIS) and positive and negative affect. Front Psychol. 2019;10:424. doi:10.3389/fpsyg.2019.00424

25. Wilborn DD, Kramer MP, Stevenson BL, Dvorak RD. Should I stay or should I go? Approach/avoidance conflict and emotional functioning. Personal Ment Health. 2018;12(4):298-308. doi:10.1002/pmh. 1428
26. Wang X, Zhang R, Chen X, et al. Psychopathological correlates and emotion regulation as mediators of approach and avoidance motivation in a chinese military sample. Front Psychiatry. 2019;10:149. doi:10.3389/fpsyt.2019.00149

27. Khosravani V, Baseri A, Kamali Z, Mohammadzadeh A, Amirinezhad A. Direct and indirect effects of behavioral inhibition/ activation systems on depression and current suicidal ideation through rumination and self-reflection. Arch Suicide Res. 2019;1-21. doi:10.1080/13811118.2019.1649224

28. Spitzer RL, Kroenke K, Williams JB, Löwe B. A brief measure for assessing generalized anxiety disorder: the GAD-7. Arch Intern Med. 2006;166(10):1092-1097. doi:10.1124/jpet.108.149989

29. Garnefski N, Kraaij V. Cognitive emotion regulation questionnaire development of a short 18-item version (CERQ-short). Pers Individ Dif. 2006;41(6):1045-1053. doi:10.1016/j.paid.2006.04.010

30. Friedman B, Heisel MJ, Delavan RL. Psychometric properties of the 15 -item geriatric depression scale in functionally impaired, cognitively intact, community-Dwelling elderly primary care patients. $J$ Am Geriatr Soc. 2010;53(9):1570-1576. doi:10.1111/j.15325415.2005.53461.x

31. Li Y, Xu Y, Chen Z. Effects of the behavioral inhibition system (BIS), behavioral activation system (BAS), and emotion regulation on depression: a one-year follow-up study in Chinese adolescents. Psychiatry Res. 2015;230(2):287-293. doi:10.1016/j. psychres.2015.09.007

32. Cai WP, Pan Y, Zhang SM, et al. Relationship between cognitive emotion regulation, social support, resilience and acute stress responses in Chinese soldiers: exploring multiple mediation model. Psychiatry Res. 2017;256:71. doi:10.1016/j.psychres.2017.06.018

33. Wang H, Hai S, Liu Y, et al. Association between depressive symptoms and sarcopenia in older Chinese community-dwelling individuals. Clin Interv Aging. 2018;13:1605. doi:10.2147/CIA. S173146

34. Sun J. Assessing goodness of fit in confirmatory factor analysis. Meas Eval Couns Dev. 2005;37(4):240-256. doi:10.1111/j.15298817.2010.00869.x

35. Schermelleh-Engel K, Moosbrugger H, Müller H. Evaluating the fit of structural equation models: tests of significance and descriptive goodness-of-fit measures. Mpr Online. 2003;8(8):23-74.

36. Mcdonald RP, Ho MHR. Principles and practice in reporting structural equation analyses. Psychol Methods. 2002;7(1):64-82. doi:10.1037/1082-989x.7.1.64

37. Corr PJ. Reinforcement sensitivity theory and personality. Neuro Biobehav Rev. 2005;28(3):317-332. doi:10.1016/j. neubiorev.2004.01.005

38. Paykel ES. Cognitive therapy and the emotional disorders: A. T. Beck. $B r \quad J$ Psychiatry. 1987;150(06):870-871. doi:10.1192/ S0007125000214918

39. Aldao A, Nolen-Hoeksema S. Specificity of cognitive emotion regulation strategies: a transdiagnostic examination. Behav Res Ther. 2010;48(10):974-983. doi:10.1016/j.brat.2010.06.002

40. Joubert C, Davidson PS, Chainay H. When do older adults show a positivity effect in emotional memory? Exp Aging Res. 2018;44 (5):455-468. doi:10.1080/0361073X.2018.1521498

41. Bares CB, Andrade F, Delva J, Grogan-Kaylor A. Personality and parenting processes associated with problem behaviors: a study of adolescents in Santiago, Chile. Soc Work Res. 2011;35(4):227-240. doi:10.1093/swr/35.4.227

42. Garnefski N, Kraaij V. The cognitive emotion regulation questionnaire. Eur $J$ Psychol Assess. 2007;23(3):141-149. doi:10.1027/1015-5759.23.3.141 


\section{Publish your work in this journal}

Psychology Research and Behavior Management is an international, peer-reviewed, open access journal focusing on the science of psychology and its application in behavior management to develop improved outcomes in the clinical, educational, sports and business arenas. Specific topics covered in the journal include: Neuroscience, memory and decision making; Behavior modification and management; Clinical applications; Business and sports performance management; Social and developmental studies; Animal studies. The manuscript management system is completely online and includes a very quick and fair peer-review system, which is all easy to use. Visit http://www. dovepress.com/testimonials.php to read real quotes from published authors. 